\title{
Neutron transmission measurements at nELBE
}

Arnd Junghans ${ }^{1, *}$, Roland Beyer ${ }^{1}$, Jürgen Claußner ${ }^{1}$, Toni Kögler ${ }^{1}$, Sebastian Urlass ${ }^{1}$, Daniel Bemmerer ${ }^{1}$, Anna Ferrari $^{1}$, Ronald Schwengner ${ }^{1}$, Andreas Wagner ${ }^{1}$, Mirco Dietz ${ }^{1,2}$, Axel Frotscher ${ }^{1,2}$, Marcel Grieger ${ }^{1,2}$, Thomas Hensel ${ }^{1,2}$, Martina Koppitz $^{1,2}$, Felix Ludwig ${ }^{1,2}$, Steffen Turkat ${ }^{1,2}$, Ralf Nolte $^{3}$, Elisa Pirovano ${ }^{1,3}$, Stefan Kopecky ${ }^{4}$, Markus Nyman $^{4}$, Arjan Plompen $^{4}$, Peter Schillebeeckx ${ }^{4}$, Erik Borris ${ }^{5}$, René Reifarth ${ }^{5}$, Daniel Veltum ${ }^{5}$, Mario Weigand ${ }^{5}$, Jan Glorius $^{6}$, Joachim Görres $^{7}$, Uwe Oberlack ${ }^{8}$, and Daniel Wenz ${ }^{8}$

${ }^{1}$ Helmholtz-Zentrum Dresden - Rossendorf, Institut für Strahlenphysik, Bautzner Landstrasse 400, 01328 Dresden, Germany

${ }^{2}$ Technische Universität Dresden, 01062 Dresden, Germany

${ }^{3}$ Physikalisch-Technische Bundesanstalt, Bundesallee 100, 38116 Braunschweig, Germany

${ }^{4}$ European Commission, Joint Research Centre, Retieseweg 111, 2440 Geel, Belgium

${ }^{5}$ Goethe Universität Frankfurt, Institut für Angewandte Physik, Max-von-Laue-Str. 1, 60438 Frankfurt am Main, Germany

${ }^{6}$ GSI Helmholtzzentrum für Schwerionenforschung GmbH, Planckstraße 1, 64291 Darmstadt, Germany

${ }^{7}$ University of Notre Dame, Department of Physics, Notre Dame, IN 46556, U.S.A.

${ }^{8}$ Johannes Gutenberg-Universität Mainz, Institut für Physik, Staudingerweg 7, 55128 Mainz, Germany

\begin{abstract}
Neutron total cross sections are an important source of experimental data in the evaluation of neutroninduced cross sections. The sum of all neutron-induced reaction cross sections can be determined with a precision of a few per cent in a relative measurement. The neutron spectrum of the photoneutron source nELBE extends in the fast region from about $100 \mathrm{keV}$ to $10 \mathrm{MeV}$ and has favourable conditions for transmission measurements due to the low instantaneous flux of neutrons and low gamma-flash background. Several materials of interest (in part included in the CIELO evaluation or on the HPRL of OECD/NEA) have been investigated: ${ }^{197} \mathrm{Au}[1,2],{ }^{\text {nat }} \mathrm{Fe}[2],{ }^{n a t} \mathrm{~W}[2],{ }^{238} \mathrm{U},{ }^{\text {nat }} \mathrm{Pt},{ }^{4} \mathrm{He},{ }^{\text {nat }} \mathrm{O},{ }^{\text {nat }} \mathrm{Ne},{ }^{\text {nat }} \mathrm{Xe}$. For gaseous targets high pressure gas cells with flat end-caps have been built that hold up to 200 bar pressure. The experimental setup will be presented including results from several transmission experiments and the data analysis leading to the total cross sections will be discussed.
\end{abstract}

\section{Introduction}

The neutron total cross section is the sum of the neutron induced partial cross sections for all open reaction channels, i.e. elastic and inelastic scattering, radiative capture, etc. It is an important experimental input for the evaluation of neutron-induced cross sections. The shape of the neutron total cross section depends on the neutron resonances of the target nuclides and on the interference with potential scattering. In the fast neutron energy range, where resonances cannot be resolved experimentally average neutron total cross sections show a large sensitivity to both compound nucleus model and optical model parameters.

The neutron total cross section can be determined in a transmission experiment, which allows a high precision of a few percent as the measurement is independent of the neutron detection efficiency. At the nELBE time of flight facility at Helmholtz-Zentrum Dresden - Rossendorf (HZDR) several transmission measurements have been conducted to determine the total neutron cross section in the fast energy range starting at $100 \mathrm{keV}$ up to about 10 $\mathrm{MeV}$. Results on ${ }^{n a t} \mathrm{Fe},{ }^{197} \mathrm{Au}$ and ${ }^{n a t} \mathrm{~W}$ have been published in ref. [2], where experimental aspects of the transmission setup and the data analysis are described in de-

*e-mail: A.Junghans@hzdr.de tail. To be able to investigate gaseous targets without corrections for the target chemical composition, high pressure gas cells with flat end-caps have been developed that stand a pressure up to 200 bar. In this paper experimental data for ${ }^{n a t} \mathrm{He},{ }^{n a t} \mathrm{O},{ }^{n a t} \mathrm{Ne},{ }^{n a t} \mathrm{Xe}$ will be presented. Furthermore, solid ${ }^{n a t} \mathrm{Pt}$, and ${ }^{238} \mathrm{U}$ (as depleted uranium) have been investigated. ${ }^{238} \mathrm{U}$ is the main component in many nuclear fuels and has also been reviewed in the international CIELO evaluation [3]. Above $5 \mathrm{MeV}$ neutron energy, total cross section measurements for a wide range of nuclides spanning the periodic table from $\mathrm{A}=1$ to 238 have been performed at LANL [4]. The data were obtained with a $1 \%$ uncertainty due to counting statistics and the uncertainty due to systematic effects is also less than $1 \%$. The neutron spectrum of nELBE allows us to extend the accessible range down to $100 \mathrm{keV}$ neutron energy in a single measurement. These data shall be compared with data from GELINA which extend from the resonance region up to $250 \mathrm{keV}$. For platinum, neutron transmission has been measured only sparsely with limited accuracy. The material is used as backing material in reference instruments and as such a good determination of the neutron transmission can be of metrological relevance [5]. The noble gases helium, neon and xenon have been investigated 
in a few experiments in the fast neutron range using quasimonoenergetic neutrons with limited energy resolution [68]. For neon the need for a more precise cross section determination has arisen from nuclear astrophysics [9] : ${ }^{17} \mathrm{O}(\alpha, n){ }^{20} \mathrm{Ne}$ can reclaim neutrons lost by ${ }^{16} \mathrm{O}(n, \gamma){ }^{17} \mathrm{O}$ in s-process scenarios. Resonances in neon transmission below $1.5 \mathrm{MeV}$ are not accessible easily in direct measurements. Xenon is used for direct dark matter detection, as of yet unknown dark matter particles scatter from xenon. To improve the understanding of the nuclear response functions of these detectors [10] a precise determination of the total cross section is necessary. Oxygen is contained in nuclear fuel and moderator or shielding materials. Precise data for the first resonance at $440 \mathrm{keV}$ are required to improve the current data evaluation [11].

\section{Experimental Set-up}

Helmholtz-Zentrum Dresden - Rossendorf operates the first photo-neutron source at a superconducting electron accelerator dedicated to measurements in the fast neutron range $[12,13]$. The floor plan of the upgraded facility is shown in Fig. 1. The electron beam is accelerated to $30 \mathrm{MeV}$ in continuous-wave mode by the superconducting electron accelerator ELBE. The micro pulse repetition rate is set to $101 \mathrm{kHz}$ with a reduced bunch charge of ca. $10 \mathrm{pC}$ for time-of-flight measurements. The electron micro pulses have a duration of only $5 \mathrm{ps}$ and thus allow for an excellent time resolution. A new compact liquid-lead circuit is utilized as a neutron-producing target. The neutron radiator consists of a Mo-tube with a rhombic cross-section and thickness of $11 \mathrm{~mm}$ through which liquid lead is pumped. The neutrons leave the neutronproducing target almost isotropically, whereas the angular distributions of electrons and bremsstrahlung are strongly forward-peaked. The collimator axis is located at an angle of 100 degrees with respect to the electron beam direction. A lead absorber of $5 \mathrm{~cm}$ thickness mounted halfway between the neutron producing target and the collimator entrance is used to suppress the bremsstrahlung intensity. The target samples are mounted in a target ladder in front of the collimator entrance at a distance of $1 \mathrm{~m}$ from the neutron producing target. The properties of the collimator and the neutron beam at the experimental area have been optimized in order to maintain the correlation of time-of-flight and neutron energy [12]. The collimator has a length of $2.5 \mathrm{~m}$ and contains three inserts of lead and borated polyethylene that are mounted inside a precision steel tube. All walls, ceiling and floor in the time-of-flight experimental hall are at least $3 \mathrm{~m}$ away from the neutron beam axis to help reduce the room return neutrons.

The neutrons were detected with a $5 \mathrm{~mm}$ thin plastic scintillator read out on both ends by high-gain PMTs Hamamatsu R2059-01. With the coincidence on both PMT signals a detection threshold of about $10 \mathrm{keV}$ neutron energy has been obtained [14]. The neutron count rate in the experiments was typically several hundred per second while the bremsstrahlung detected had a count rate of a few thousand per second. A typical time-of-flight spectrum with a neon sample in the beam is shown in Fig. 2.

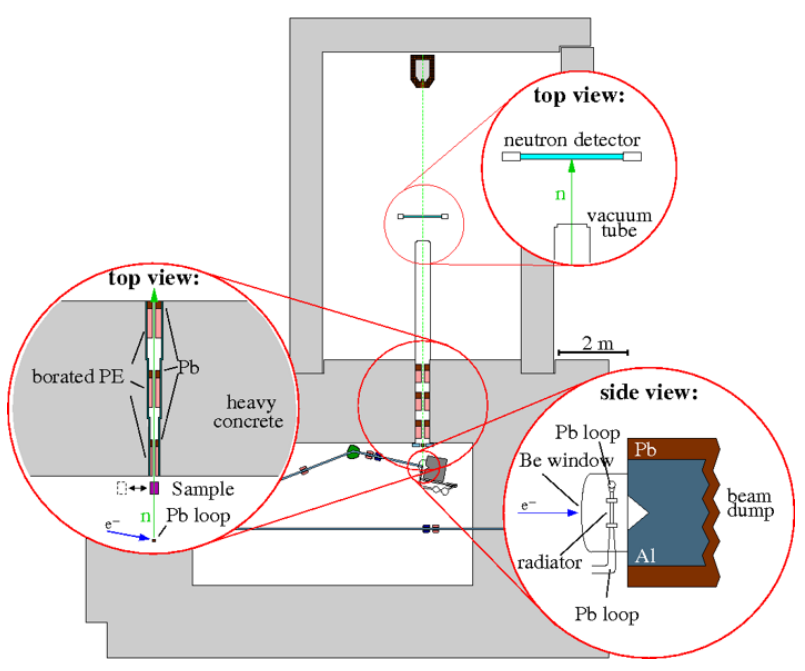

Figure 1. Floor plan of the neutron time-of-flight facility nELBE at HZDR. The neutrons are produced by the electron beam hitting a liquid lead circuit as neutron producing target, see inset on the lower right. The neutron beam is shaped by a collimator and guided to the neutron time-of-flight hall, see inset on the left. The detection setup is located in the time-of-flight hall, see upper inset. For neutron transmission experiments a plastic scintillator with a low threshold for recoil proton signals $\left(E_{n}>10\right.$ $\mathrm{keV}$ ) is used. The transmission samples are located in a movable absorber ladder in the front of the collimator.

The flight path was $918 \mathrm{~cm}$ long. A narrow gate was used on the time difference spectrum of the two PMTs to reduce detection of double hits. A dead time of $3 \mu$ s was inserted after each coincidence hit to efficiently suppress PMT afterpulses that mainly arise from the gamma-flash.

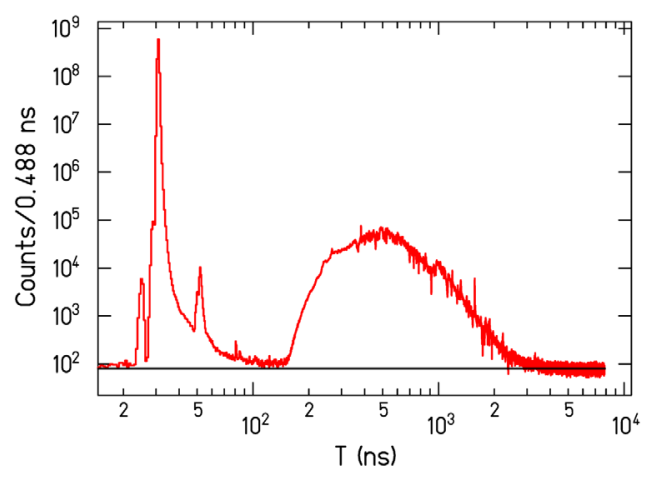

Figure 2. Deadtime corrected time-of-flight spectrum with a ${ }^{\text {nat }} \mathrm{Ne}$ sample in the beam. PMT after-pulses are suppressed as described in the text. The small first peak at $25 \mathrm{~ns}$ is due to double hits in the scintillator. The bremsstrahlung peak is located at $30.6 \mathrm{~ns}$, its width is $0.61 \mathrm{~ns}$ (FWHM). The broader peak at $52 \mathrm{~ns}$ is due to bremsstrahlung backscattered from the beam dump and rear wall. The neutron time-of-flight range is from about 150 to $2500 \mathrm{~ns}$. The black line is the result of a fit of a constant random background rate. 
The transmission measurements of gaseous target samples were made using high-pressure gas cells made from stainless steel tubing with flat end-caps. A cross section cut of the gas cell is shown in Fig. 3. To cancel the transmission factor through the end caps an identical evacuated cell was used in the "target out of beam" measurements.

The target areal densities were determined by measuring the temperature and pressure during and after the filling procedure of the gas targets using a pressure transducer with an absolute accuracy of 1.25 bar (For the oxygen and xenon experiments a high-precision pressure transducer was used with an absolute accuracy of 0.125 bar.) Only new high-purity gas bottles were used with a purity of the gases of 99.99 per cent or higher. The fluid equation of state used to convert the measured pressure and temperature to density was taken from the NIST data base [15]. The metallic target samples ${ }^{n a t} \mathrm{Pt}$, and ${ }^{238} \mathrm{U}$ (as depleted uranium) had a right cylindrical shape with a diameter of $25 \mathrm{~mm}(\mathrm{Pt})$ and $50.8 \mathrm{~mm}(\mathrm{U})$. The uranium target used here was from pure depleted uranium. The isotopic depletion has been verified by gamma-spectroscopy. The oxide layer was thin and the target samples were encased in a thin aluminum housing to allow safe handling. An identical empty housing was used in the "target out" measurements. The target properties are listed in Table 1.

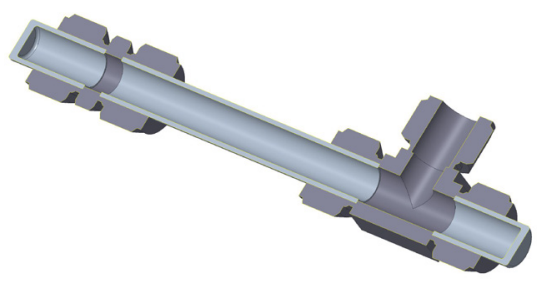

Figure 3. Cross section cut of a high pressure gas cell used for the neutron transmission measurements with gaseous targets. The cell is made from stainless steel with cylindrical end-caps having a wall thickness of $3 \mathrm{~mm}$ using standard Swagelock components. The maximum pressure is up to 200 bar. The length of the gas volume is $393 \mathrm{~mm}$. The tube is made from $30 \times 3$ $\mathrm{mm}$ stainless steel. Not shown are the valve and pressure gauge connected on the open ended flange.

\section{Data analysis}

The neutron transmission $T_{\text {exp }}\left(t_{i}\right)$ is determined from the ratio of the background and deadtime corrected count rates with sample in the beam to sample out of the beam as a
Table 1. Target composition, purity (gases in volume per cent, metals in weight per cent) and atomic areal density in atoms/barn.

\begin{tabular}{lll}
\hline${ }^{n a t} \mathrm{He}$ & 99.999 & $0.1797(11)$ \\
${ }^{n a t} \mathrm{O}$ & 99.998 & $0.09191(60)$ \\
${ }^{n a t} \mathrm{Ne}$ & 99.99 & $0.1624(11)$ \\
${ }^{n a t} \mathrm{Xe}$ & 99.998 & $0.07762(50)$ \\
${ }^{n a t} \mathrm{Pt}$ & 99.95 & $0.09290(22)$ \\
${ }^{238} \mathrm{U}$ & 99.7 & $0.05450(6)$ \\
\hline
\end{tabular}

function of neutron time-of-flight channel $t_{i}$ :

$$
\begin{aligned}
T_{\exp }\left(t_{i}\right)=\frac{\sum_{k}\left(N_{\text {in }, k}\left(t_{i}\right)-B_{\text {in }, k}\left(t_{i}\right)\right) f_{\text {in }, k}}{\sum_{k} t_{\text {real }, \text { in }, k}} \\
\cdot \frac{\sum_{k} t_{\text {real }, \text { out }, k}}{\sum_{k}\left(N_{\text {out }, k}\left(t_{i}\right)-B_{\text {out }, k}\left(t_{i}\right)\right) f_{\text {out }, k}}
\end{aligned}
$$

where $N_{\text {in/out }}\left(t_{i}\right)$ and $B_{\text {in/out }}\left(t_{i}\right)$ are the deadtime corrected numbers of detected events and level of background events in each time-of-flight channel $i$ with and without the target in the beam. The real times for each run $k$ with target in/out are denoted by $t_{\text {real,in }, k}, t_{\text {real,out, } k}$ The dead-time correction is time-of-flight dependent, as explained in ref.[2]. The relative live times were in a range from $0.88(\mathrm{O})$ and 0.98 (Pt) for "target out of the beam" measurements and 0.865 (O) to $0.935(\mathrm{Pt})$ for "target in beam" measurements. The experiments were done by periodically moving the target sample in and out of the beam with counting times adapted to maximize the statistics. Typical counting times for each setting were 10-20 min. The experiments were done with about 100-150 hours of beam time. Listmode data were recorded in separate runs of about 2-4 hours. The transmission was calculated using the sum of all these runs $k$ where no beam intensity fluctuation or failure occurred in the "target in" and "target out" settings. The factors $f_{\text {in }, k}$ and $f_{\text {out }, k}$ are normalization factors to correct for remaining fluctuations in the neutron source intensity:

$$
\begin{aligned}
& f_{\text {out }, k}=\frac{t_{\text {real }, \text { out }, k}}{\sum_{i}\left(N_{\text {out }, k}\left(t_{i}\right)-B_{\text {out }, k}\left(t_{i}\right)\right)} \\
& f_{\text {in }, k}=\langle T\rangle \frac{t_{\text {real }, \text { in }, k}}{\sum_{i}\left(N_{\text {in }, k}\left(t_{i}\right)-B_{\text {in }, k}\left(t_{i}\right)\right)}
\end{aligned}
$$

The neutron count rate for each run $k$ with target out of the beam and summed over the full neutron time-of-flight range $i$ is used as a neutron source intensity monitor in eq.(2). For the "target in" settings the neutron source intensity monitor is approximated with the target-in count rate divided by the mean integral transmission factor $\langle T\rangle$ over the full neutron time-of-flight range $i$ of the full experiment including all runs $k$.

From the measured transmission as a function of timeof-flight $T_{\exp }\left(E_{\mathrm{n}}\left(t_{i}\right)\right)$ an effective neutron total cross section $\left\langle\sigma_{\text {tot }}\left(E_{\mathrm{n}}\right)\right\rangle$ can be determined:

$$
\left\langle\sigma_{\text {tot }}\left(E_{\mathrm{n}}\right)\right\rangle=-\frac{1}{n} \ln T_{\exp }
$$

where $n$ is the atomic areal density of the target sample. To determine the neutron transmission and the total cross 
section from the measured time-of-flight distribution with a relative accuracy of a few percent several corrections are to be carried out:

1. Correction for a time-of-flight dependent dead time

2. Subtraction of a random background in the time-offlight spectra

3. Correction for fluctuations of the neutron-beam intensity

4. Correction for in-scattering of neutrons

5. Correction for resonant self-shielding in thick transmission samples

Random background and dead-time corrections are important at low and high neutron energy, where the neutron source intensity is already decreasing. In these transmission experiments a low beam intensity and a very compact neutron producing target without any materials that would slow down neutrons were used. The random background can be described by a constant value in time-of-flight. It is mostly dominated by random coincidences due to ambient natural radioactivity whereas the room return background of neutrons is relatively low [1]. Fig. 2 shows a typical background rate. The determination of the background levels $B_{\text {in/out }}$ is done by calculating the mean bin content in the time-of-flight ranges before the $\gamma$-flash and between 5 to $9 \mu \mathrm{s}$. In the time-of-flight region around $150 \mathrm{~ns}$ before the first fast neutrons arrive the background level is higher than estimated from the mean bin content between 5 to 9 $\mu \mathrm{s}$. This background tail is due to insufficient suppression of PMT after-pulses mostly from the gamma-flash. Due to the low background-to-total ratio it has been neglected in the transmission determination [2]. The background-tototal ratio is shown in Fig. 4.

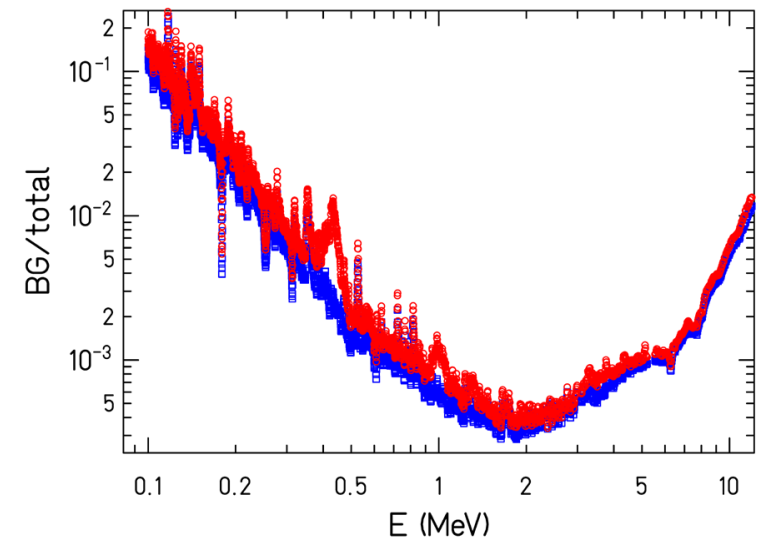

Figure 4. The background-to-total ratio shown for the oxygen experiment. The red circles denote the ratio with the oxygen gas cell in the beam. The blue squares denote the ratio with the evacuated gas cell in the beam.

The correction for beam fluctuations has been described above with eqs. $(1,2,3)$. The relative uncertainties of the total cross sections estimated from the fluctuations of the transmission values $k$ in each experiment are around $1.5 \%$. Fig. 5 shows the fluctuations of the neutron beam intensity of the oxygen experiment, where the beam was stable. In the xenon experiment a reduced beam stability led to a larger uncertainty of $3 \%$ in the cross section. The total cross section determined by the sum of the runs $k$ as described above differed by less than $0.5 \%$ from the total cross section determined with the weighted average of the transmissions of the runs $k$.

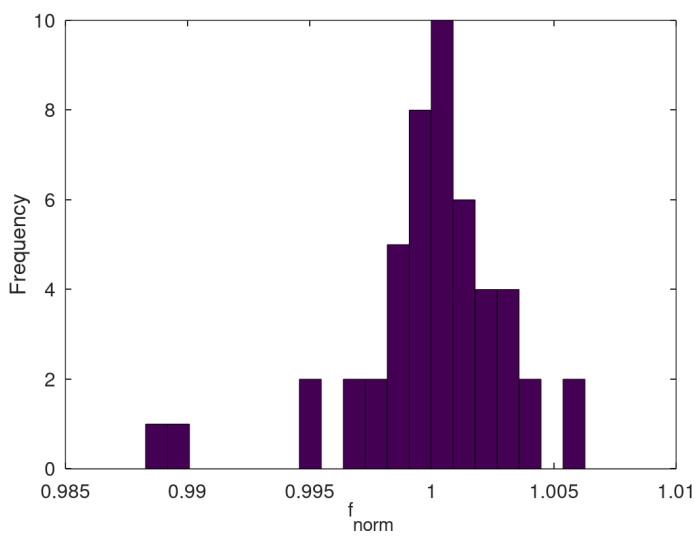

Figure 5. The neutron source intensity fluctuations shown for the oxygen experiment. The quantity $f_{\text {norm }}$ shown in the histogram is the ratio of eqs.(3),(2).

In-scattering of neutrons was minimized by the geometry of the setup: The collimator strongly limits the solid angle under which neutrons can be registered and only neutrons passing through the full length of the sample can hit the detector. The probability for multiple in-scattering of fast neutrons has been found to be less than $0.1 \%$.

The resonance self-shielding correction for thick samples in the unresolved resonance range can be obtained in a statistical way by using the probability table method with MCNP, for a description see e.g. ref. [2]. The data shown here were not corrected for this effect, which is typically smaller than $1 \%$ in total cross section for neutron energies above $150-200 \mathrm{keV}$.

\section{Results}

The neutron total cross sections of ${ }^{n a t} \mathrm{He},{ }^{n a t} \mathrm{O},{ }^{n a t} \mathrm{Ne}$, ${ }^{n a t} \mathrm{Xe},{ }^{n a t} \mathrm{Pt}$, and ${ }^{238} \mathrm{U}$ have been measured with the nELBE time-of-flight facility at HZDR in the energy range from about 0.10 to $10 \mathrm{MeV}$, see Fig. 6. The energy resolution $\Delta E / E$ increases in this energy range from $2 \cdot 10^{-3}$ to $8 \cdot 10^{-3}$ (FWHM). The resolution has been improved by reducing the scintillation detector thickness as compared to previous measurements $[1,2]$. This resolution is sufficient to resolve low lying resonances in light nuclei and for average cross sections that can be compared with optical model calculations. For helium, neon and xenon only a few measurements exist. Our helium data are in good agreement with the measurement by Goulding et al., [8] and extend the measured energy range to lower energies. For neon and xenon only data from quasimonoener- 


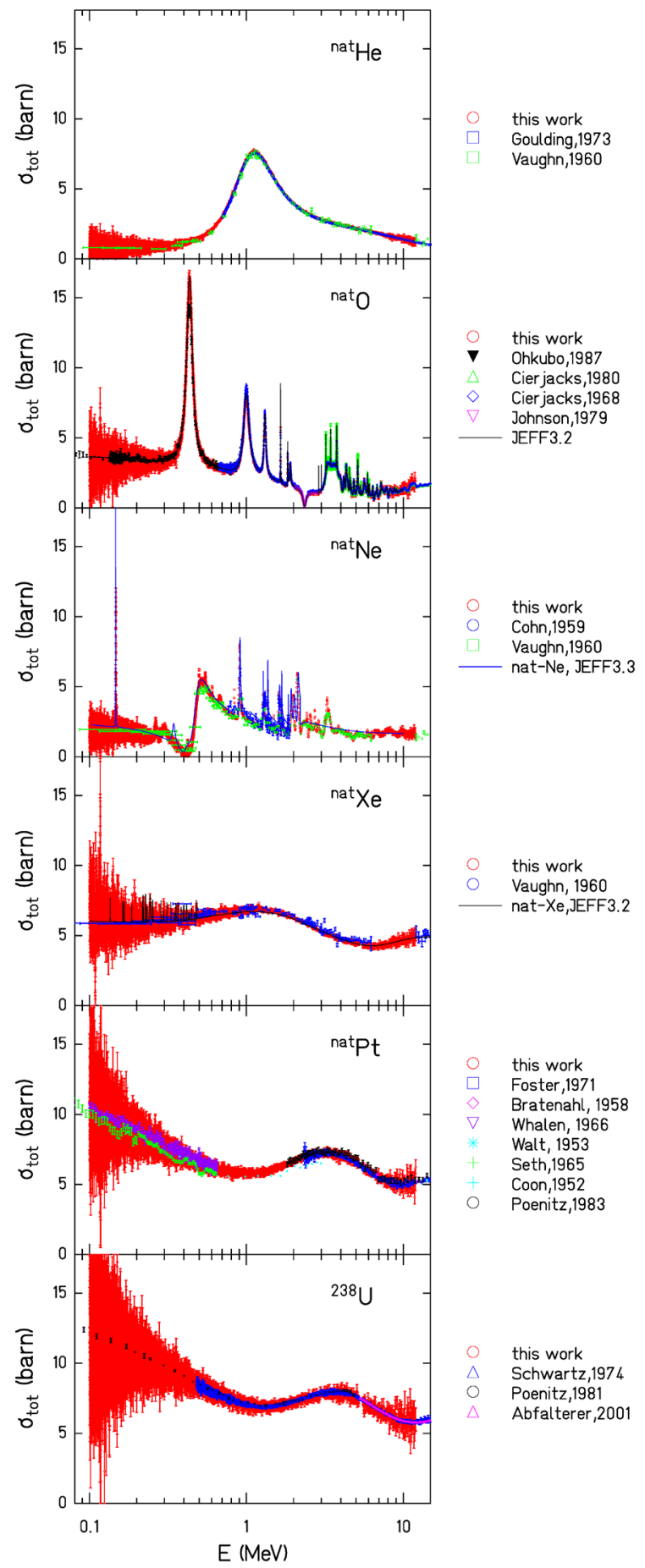

Figure 6. The effective total cross sections for ${ }^{n a t} \mathrm{He},{ }^{\text {nat }} \mathrm{O},{ }^{n a t} \mathrm{Ne}$, ${ }^{n a t} \mathrm{Xe},{ }^{\text {nat }} \mathrm{Pt}$, and ${ }^{238} \mathrm{U}$ are shown in comparison with selected experimental results from literature $[4,6-8,16-28]$ taken from the EXFOR data base [29] and data from the JEFF evaluation [30]. The data from this work are shown with energy bin sizes corresponding to a time-of-flight bin size of $0.5 \mathrm{~ns}$.

getic sources exist with a limited energy resolution [6, 7]. Our new high-resolution data show several resonances not seen before. The xenon data should help to improve optical model parametrisation, e.g.[31]. The first resonance in oxygen has been measured with high statistical accuracy of 1-2 \% for a time-of-flight bin size of $0.5 \mathrm{~ns}$ and good en- ergy resolution. The minimum transmission value at this resonance was $0.215(2)$, while the maximum transmission was $1.002(4)$ at the cross section dip at $2.35 \mathrm{MeV}$. Our data below the first resonance in oxygen are slightly below the measurement of Ohkubo et al. [16] but do not require a correction for moisture content of the target. The platinum data are in good agreement with earlier results and the first experiment to cover a large neutron energy range. The dip at $1 \mathrm{MeV}$ in the excitation function was not measured up to now. For ${ }^{238} \mathrm{U}$ our data allow us to make a connection from low energies to the precise measurements from Abfalterer et al. [4] that extend from a neutron energy of 5 $\mathrm{MeV}$ to several hundred $\mathrm{MeV}$ neutron energy.

\section{Acknowledgments}

We thank the ELBE accelerator crew for providing stable beam operation as well as Andreas Hartmann and Maik Görler for excellent continuing technical support. This work was supported by the German Federal Ministry for Education and Science (TRAKULA project, contract number 02NUK13A) and by the European Commission within the Seventh Framework Programme through Fission-2013-CHANDA (project number 605203).

\section{References}

[1] R. Hannaske, Z. Elekes, R. Beyer, A. Junghans, D. Bemmerer, E. Birgersson, A. Ferrari, E. Grosse, M. Kempe, T. Kögler et al., The European Physical Journal A 49, 137 (2013)

[2] R. Beyer, A.R. Junghans, P. Schillebeeckx, I. Sirakov, T.Y. Song, D. Bemmerer, R. Capote, A. Ferrari, A. Hartmann, R. Hannaske et al., The European Physical Journal A 54, 81 (2018)

[3] R. Capote, A. Trkov, EPJ Nuclear Sciences \& Technologies 4, 27 (2018)

[4] W.P. Abfalterer, F.B. Bateman, F.S. Dietrich, R.W. Finlay, R.C. Haight, G.L. Morgan, Physical Review C 63, 044608 (2001)

[5] R. Nolte, M.S. Allie, F.D. Brooks, A. Buffler, V. Dangendorf, J.P. Meulders, H. Schuhmacher, F.D. Smit, M. Weierganz, Nuclear Science and Engineering 156, 197 (2007)

[6] H.O. Cohn, J.L. Fowler, Physical Review 114, 194 (1959)

[7] F.J. Vaughn, W.L. Imhof, R.G. Johnson, M. Walt, Physical Review 118, 683 (1960)

[8] C. Goulding, P. Stoler, J. Seagrave, Nuclear Physics A 215, 253 (1973)

[9] A. Best, S. Falahat, J. Görres, M. Couder, R. deBoer, R.T. Güray, A. Kontos, K.L. Kratz, P.J. LeBlanc, Q. Li et al., Physical Review C 87, 045805 (2013)

[10] B. Beskers, A small dual-phase xenon TPC with $A P D$ and PMT readout for the study of liquid xenon scintillation, in Proceedings of Technology and Instrumentation in Particle Physics 2014 PoS(TIPP2014) (Sissa Medialab, 2015), p. 160 
[11] L. Leal, E. Ivanov, G. Noguere, A. Plompen, S. Kopecky, EPJ Nuclear Sciences \& Technologies 2, 43 (2016)

[12] J. Klug, E. Altstadt, C. Beckert, R. Beyer, H. Freiesleben, V. Galindo, E. Grosse, A. Junghans, D. Légrády, B. Naumann et al., Nuclear Instruments and Methods in Physics Research Section A: Accelerators, Spectrometers, Detectors and Associated Equipment 577, 641 (2007)

[13] R. Beyer, E. Birgersson, Z. Elekes, A. Ferrari, E. Grosse, R. Hannaske, A. Junghans, T. Kögler, R. Massarczyk, A. Matić et al., Nuclear Instruments and Methods in Physics Research Section A: Accelerators, Spectrometers, Detectors and Associated Equipment 723, 151 (2013)

[14] R. Beyer, E. Grosse, K. Heidel, J. Hutsch, A. Junghans, J. Klug, D. Légrády, R. Nolte, S. Röttger, M. Sobiella et al., Nuclear Instruments and Methods in Physics Research Section A: Accelerators, Spectrometers, Detectors and Associated Equipment 575, 449 (2007)

[15] E.W. Lemmon, M.O. McLinden, D.G. Friend, Thermophysical Properties of Fluid Systems, Vol. NIST Chemistry WebBook, NIST Standard Reference Database Number 69, (National Institute of Standards and Technology, Gaithersburg MD, 20899, 2016), https: //webbook.nist.gov/ chemistry/

[16] M. Ohkubo, Tech. Rep. JAERI-M-86-193, JAERI (1987)

[17] S. Cierjacks, F. Hinterberger, G. Schmalz, D. Erbe, P. Rossen, B. Leugers, Nuclear Instruments and Methods 169, 185 (1980)

[18] S. Cierjacks, P. Forti, D. Kopsch, L. Kropp, J. Nebe, H. Unseld, Tech. Rep. KFK 1000, Kernforschungszentrum Karlsruhe (1968), https://publikationen.bibliothek.kit. edu/270002514

[19] C. Johnson, J. Fowler, N. Hill, J. Ortolf, Measurement of the 2.35-MeV window in $O+n$, in Proc.Intern.Conf. on Nuclear Cross Section for Technology, Knoxville, 1979 (1979), NBS-SP-594 (1980), p. 807

[20] D.G. Foster, D.W. Glasgow, Physical Review C 3, 576 (1971)

[21] A. Bratenahl, J.M. Peterson, J.P. Stoering, Physical Review 110, 927 (1958)

[22] J. Whalen, W. Meadows, Tech. Rep. ANL7210,16,1966, Argonne National Laboratory (1966)

[23] M. Walt, R.L. Becker, A. Okazaki, R.E. Fields, Physical Review 89, 1271 (1953)

[24] K. Seth, Physics Letters 16, 306 (1965)

[25] J.H. Coon, E.R. Graves, H.H. Barschall, Physical Review 88, 562 (1952)

[26] W. Poenitz, J. Whalen, Tech. Rep. ANL/NDM80, Argonne National Laboratory (1983), https://www.ne.anl.gov/capabilities/ nd/reports/ANLNDM61.html

[27] R.B. Schwartz, R.A. Schrack, H.T. Heaton, Nuclear Science and Engineering 54, 322 (1974)

[28] W.P. Poenitz, J.F. Whalen, A.B. Smith, Nuclear Science and Engineering 78, 333 (1981)

[29] N. Otsuka, Progress in International Collaboration on the EXFOR Library, in Proc. Int. Conf. Nuclear Data for Science and Technology Beijing (2019)

[30] A. Plompen, The Joint Evaluated Fission and Fusion (JEFF) Nuclear Data Library, in Proc. Int. Conf. Nuclear Data for Science and Technology Beijing (2019)

[31] G. Noguere, O. Litaize, P. Archier, C. De Saint Jean, P. Mutti, Nuclear Physics A 870-871, 131 (2011) 